\title{
Fractal dimensions of wildfire spreading
}

\author{
S.-L. Wang, H.-I. Lee, and S.-P. Li \\ Institute of Physics, Academia Sinica 128 Sec. 2, Academia Rd., Nankang, Taipei, 11529, Taiwan (ROC) \\ Correspondence to: S.-L. Wang (r96222073@ntu.edu.tw)
}

Received: 2 December 2013 - Revised: 22 April 2014 - Accepted: 24 June 2014 - Published: 7 August 2014

\begin{abstract}
The time series data of 31 wildfires in 2012 in the US were analyzed. The fractal dimensions (FD) of the wildfires during spreading were studied and their geological features were identified. A growth model based on the cellular automata method is proposed here. Numerical study was performed and is shown to give good agreement with the fractal dimensions and scaling behaviors of the corresponding empirical data.
\end{abstract}

\section{Introduction}

Wildfire is one of the main disturbances in an ecosystem and has been of much concern in many countries over the years. Historically, it has been the cause of numerous and possibly irreversible damages, with deep ecological as well as sociological and economic impacts. In some extreme cases, the whole ecosystem might be wiped out and result in large numbers of human casualties. It is therefore understood that the need for designing and developing effective ways of dealing with wildfires is constantly increasing, as such phenomena appear ever more often. In fact, the area burned annually by wildfire has always been a factor that influences policy decisions and future land-use planning in countries such as the United States and Canada. There are indeed many factors that can affect the spreading of wildfire and the area that will be burned once the fire has started. Among them, climate (Kitzberger et al., 2007), forest type (McKenzie et al., 1996), wind (Weise and Biging, 1996), landscape (Romme, 1982) and also human activities are some of the major factors that can influence the occurrence and spreading of wildfires. Among these factors, climate seems to be a major factor that governs the rate of spread and the burned area of wildfires. For example, there are significantly more cases of wildfires reported during summer time, when the temperature in a region is considerably higher. Early wildfire history evidence from diverse climate regions and vegetation also suggests that ignitions and spreads of wildfires have strong relations to climate changes (Matthew et al., 2010; Littell et al., 2009; Westerling and Bryant, 2008). In recent years, there have also appeared studies that investigate the relationship between global and regional warming in wildfire activities (Westerling et al., 2006; Lee et al., 2013). Over the years, there have been many studies on the modeling of wildfire spreading by various approaches (Sullivan, 2009a) to assist policy makers in designing and developing effective ways of dealing with wildfires. In recent years, it has also caught the attention of physicists, who want to understand this subject from the physical point of view (Bak et al., 1990; Chen et al., 1990). In the study of complex dynamical systems such as wildfires, physicists are interested in investigating whether they are critical systems. In these studies, the authors modeled the wildfires as self-organized critical systems and studied their properties. A more interesting feature of wildfires is that satellite images show that the spreading of wildfires looks fractal (Caldarelli et al., 2001). A natural approach would therefore be to use some physical models such as percolation models (Caldarelli et al., 2001) to mimic the spreading of wildfires. One would then be able to calculate the statistical properties of wildfires by using these physical models and comparing them with empirical data. Previous works (Caldarelli et al., 2001) only analyzed the final image of wildfires because of data availability. With the advancement of modern technology, more and better wildfire image data are now available. Some of the large wildfires can now be recorded daily once they are discovered, and one can indeed observe the spreading of a particular wildfire as a function of time. The images have a spatial resolution of $5 \mathrm{~m}$, and thus provide much more accurate data for analysis. One would then be able to analyze the dynamical and statistical properties of wildfires with better precision. 
Table 1. The list of 31 wildfires analyzed in the paper.

\begin{tabular}{|c|c|c|c|c|c|}
\hline Name & $\begin{array}{c}\text { Date } \\
(\mathrm{mm} / \mathrm{dd})\end{array}$ & $\begin{array}{c}\text { Position } \\
(\mathrm{W}, \mathrm{N})\end{array}$ & $\begin{array}{c}\text { State } \\
\text { (USA) }\end{array}$ & $\begin{array}{c}\text { Size } \\
\left(\mathrm{km}^{2}\right)\end{array}$ & $\begin{array}{c}\text { Duration } \\
\text { (days) }\end{array}$ \\
\hline County Line & $04 / 05$ & $82.5,30.5$ & FL & 141.5 & 17.7 \\
\hline Sunflower & $05 / 13$ & $111.5,34.0$ & $\mathrm{AZ}$ & 65.2 & 8.7 \\
\hline Gladiator & $05 / 14$ & $112.3,34.2$ & $\mathrm{AZ}$ & 61.8 & 7.5 \\
\hline Little Sand & $05 / 24$ & $107.2,37.4$ & $\mathrm{CO}$ & 100.9 & 43.3 \\
\hline High Park & 06/09 & $105.4,40.6$ & $\mathrm{CO}$ & 354.3 & 20.0 \\
\hline Little Bear & 06/09 & $105.8,33.4$ & NM & 179.8 & 17.0 \\
\hline Poco & $06 / 15$ & $110.9,34.1$ & $\mathrm{AZ}$ & 48.4 & 7.2 \\
\hline Russells Camp & $06 / 19$ & $105.8,42.5$ & WY & 22.1 & 9.9 \\
\hline Waldo Canyon & $06 / 23$ & $104.9,38.9$ & $\mathrm{CO}$ & 73.9 & 12.0 \\
\hline Weber & $06 / 23$ & $108.3,37.3$ & $\mathrm{CO}$ & 41.0 & 9.0 \\
\hline Wood Hollow & $06 / 25$ & $111.5,39.7$ & UT & 192.0 & 4.2 \\
\hline \multicolumn{6}{|l|}{ Neighbor } \\
\hline Mountain & $06 / 26$ & $78.4,38.7$ & VA & 8.6 & 9.3 \\
\hline Point & $06 / 26$ & $78.3,38.9$ & VA & 4.7 & 7.7 \\
\hline Ash Creek & $06 / 27$ & $106.5,45.7$ & MT & 1005.2 & 7.5 \\
\hline Seeley & $06 / 27$ & $111.2,39.5$ & UT & 192.7 & 14.2 \\
\hline Arapaho & $06 / 28$ & $105.5,42.2$ & WY & 396.7 & 13.4 \\
\hline Clay Springs & $06 / 28$ & $112.4,39.3$ & UT & 436.5 & 11.4 \\
\hline Wolf Den & $06 / 30$ & $109.2,39.8$ & UT & 80.4 & 7.0 \\
\hline Shingle & $07 / 03$ & $112.6,37.5$ & UT & 32.8 & 7.1 \\
\hline Mill & $07 / 08$ & $122.7,39.3$ & $\mathrm{CA}$ & 119.5 & 12.8 \\
\hline \multicolumn{6}{|l|}{ Miller } \\
\hline Homestead & $07 / 10$ & $119.2,42.8$ & OR & 647.1 & 7.2 \\
\hline Chrandal Creek & $07 / 11$ & $114.2,45.5$ & MT/ID & 10.1 & 20.0 \\
\hline Flat & $07 / 12$ & $123.4,40.8$ & $\mathrm{CA}$ & 7.0 & 6.5 \\
\hline Robbers & $07 / 12$ & $120.9,39.0$ & $\mathrm{CA}$ & 10.7 & 8.5 \\
\hline Chips & $07 / 30$ & $121.3,40.0$ & $\mathrm{CA}$ & 305.3 & 37.7 \\
\hline Trinity Ridge & $08 / 04$ & $115.4,43.7$ & ID & 593.5 & 64.0 \\
\hline Barry Point & $08 / 07$ & $120.8,42.1$ & OR & 376.0 & 27.4 \\
\hline Merino & $08 / 12$ & $114.9,44.7$ & ID & 31.9 & 37.0 \\
\hline Cascade Creek & 09/01 & $121.5,46.1$ & WA & 81.2 & 36.0 \\
\hline Sheep & $09 / 07$ & $116.3,45.5$ & ID & 196.7 & 33.7 \\
\hline Pole Creek & $09 / 11$ & $121.6,44.2$ & OR & 108.4 & 25.9 \\
\hline
\end{tabular}

In this paper, we provide the first analysis of wildfire spreading time series. We show that wildfire spreading exhibits a universal scaling behavior that has never been reported before. We also show that the satellite images reveal very interesting statistical and dynamical properties as the wildfire spreads.

\section{Analysis of empirical data}

We construct here the digital perimeters of the wildfire events that happened in America that are available from the Geospatial Multi-Agency Coordination (Geomac) database (Geomac, 2012) as a form of geographic information system (GIS). In the Geomac database, these digital data are reconstructed from Global Positioning System (GPS) and infrared imagery. We analyze a total of 31 wildfires that either spread over a large area or persisted for a long enough time before they were extinguished. For example, the wildfire that occurred in Ash Creek, Montana had the largest burned area of $1005 \mathrm{~km}^{2}$, and the wildfire in Trinity Ridge, Idaho persisted for a total of 64 days. Table 1 above is the list of wildfires analyzed in this paper.

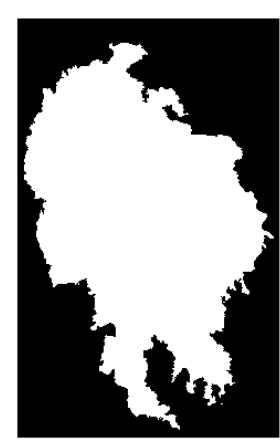

(a)

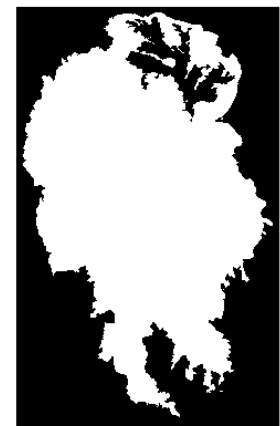

(d)

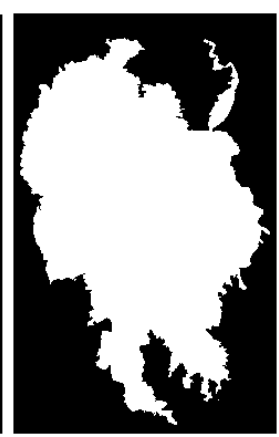

(b)

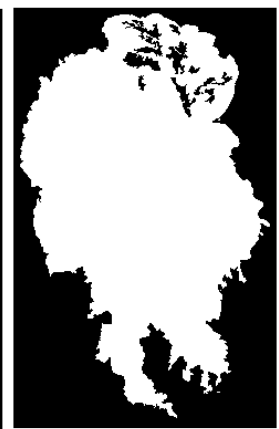

(e)

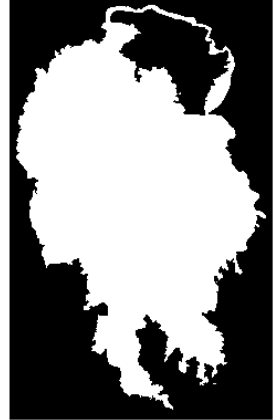

(c)

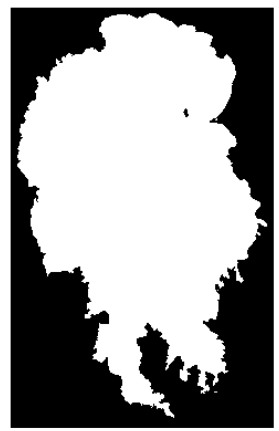

(f)
Figure 1. The time sequence of the Little Bear wildfire in New Mexico, USA in 2012: (a) 18 June, 21:04 LT, (b) 19 June, 13:33 LT, (c) 19 June, 21:42 LT, (d) 20 June, 08:42 LT, (e) 21 June, 20:57 LT, (f) 22 June, 20:46 LT. Each of the panels (a)-(f) has a width of 2750 pixels and a height of 4450 pixels, corresponding to an actual size of $13.75 \mathrm{~km} \times 22.25 \mathrm{~km}$.

In the empirical wildfire data sets, both the burned and burning sites are given by their longitudinal and latitudinal coordinates. To construct the digital perimeters of the wildfire events, we first project the empirical wildfire data recorded in geographic coordinates onto a two-dimensional square lattice, transforming the wildfire data into digital graphs. Each lattice site corresponds to one pixel of the digital image of the wildfire from the database, with a precision of $25 \mathrm{~m}^{2}$ per pixel. Figure 1 is an example of a wildfire that occurred in Little Bear, New Mexico, USA in 2012 after we project its geographic coordinate onto a two-dimensional lattice. The images correspond to a time sequence: (a) 18 June, 21:04 LT, (b) 19 June, 13:33 LT, (c) 19 June, 21:42 LT, (d) 20 June, 08:42 LT, (e) 21 June, 20:57 LT, and (f) 22 June, 20:46 LT. The white portion in each figure corresponds to the burned area, while the black portion is the unburned surroundings. Each of the panels (a)-(f) in Fig. 1 has a width of 2750 pixels and a height of 4450 pixels, corresponding to an actual size of $13.75 \mathrm{~km} \times 22.25 \mathrm{~km}$.

From the digital graph, we can compute the burned area $(A)$ of the wildfire under study. To proceed, we label lattice sites with 1 for the burned sites and 0 for the unburned sites. Figure 2 is an illustration of a digital graph of wildfire, with 1 for the burned sites and 0 for the unburned 


\begin{tabular}{|l|l|l|l|l|l|l|}
\hline 0 & 0 & 0 & 0 & 0 & 0 & 0 \\
\hline 0 & 0 & 1 & 1 & 0 & 0 & 0 \\
\hline 0 & 1 & 1 & 1 & 1 & 1 & 0 \\
\hline 0 & 0 & 1 & 1 & 1 & 1 & 0 \\
\hline 0 & 1 & 1 & 1 & 1 & 0 & 0 \\
\hline 0 & 1 & 1 & 0 & 1 & 1 & 0 \\
\hline 0 & 0 & 0 & 0 & 0 & 0 & 0 \\
\hline
\end{tabular}

Figure 2. Example of a binary graph. For this graph, $A=19$, and its accessible perimeter $L=18$.

sites. By counting the number of lattice sites with value 1 , we will obtain an approximate value of the burned area of the wildfire. We will then deduce the radius of gyration $\left(R_{\mathrm{g}}\right)$ from its burned area. The radius of gyration of the burned area can be obtained as follows: let the location of the $i$ th site of the burned area be $\left(x_{i}, y_{i}\right)$. The location of the center of the burned area is then equal to $\left(x_{\mathrm{rc}}, y_{\mathrm{rc}}\right)=$ $\left(\sum_{i} x_{i} / A, \sum_{i} y_{i} / A\right)$, and the radius of gyration would then be equal to $\sqrt{\left(\sum_{i}\left(x_{i}^{2}+y_{i}^{2}\right) / A-\left(x_{\mathrm{rc}}^{2}+y_{\mathrm{rc}}^{2}\right)\right.}$.

In a straightforward way, one can also calculate the accessible perimeter $(L)$ of the wildfire as it spreads. We compute here the accessible perimeter in the following way. In the original digital graph, when a burned site (1) has all eight nearest and next-nearest neighbors as burned sites (1), we will change its label to 0 . After we do this, we can count the number of $1 \mathrm{~s}$ in the new graph and obtain the accessible perimeter $(L)$ of the wildfire. Figure 3 is an illustration of this procedure. In Fig. 3a, the sites in yellow and red are burned sites, but only the red site is surrounded by burned sites as its nearest and next-nearest neighbors. We then re-label this site as 0 , as shown in Fig. 3b. After we carry out this relabeling, we will then count the sites labeled 1 to obtain $L$. One can of course make the perimeter measurement in other ways. As an example, let us again refer to Fig. 3a. One may neglect the corner sites (yellow) in the neighborhood of the red site. Let us take the upper left corner site as an illustration. In this case, we neglect the site, but instead take the length of the perimeter around this yellow corner site as the length of the distance of the centers on the left and at the top of this corner site. The perimeter around this corner would then be $2 \sqrt{2}$ instead of 3 . This would give an error of about $5 \%$. The difference by using these two different ways of counting would differ by much less than $5 \%$. We should mention here that the difference by using different counting methods

\begin{tabular}{|l|l|l|l|l|}
\hline 0 & 1 & 1 & 0 & 0 \\
\hline 1 & 1 & 1 & 1 & 1 \\
\hline 0 & 1 & 1 & 1 & 1 \\
\hline 1 & 1 & 1 & 1 & 0 \\
\hline 1 & 1 & 0 & 1 & 1 \\
\hline
\end{tabular}

(a)

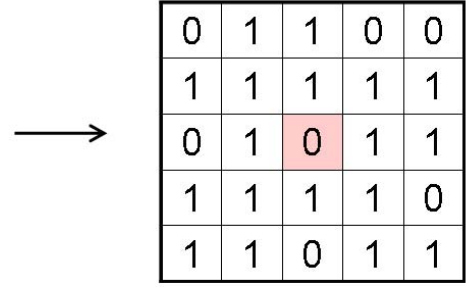

(b)
Figure 3. An illustration of the evaluation of $L$. In (a), the sites in yellow and red are burned sites (1), but only the red site is surrounded by burned sites as its nearest and next-nearest neighbors. We re-label this site as 0 , as shown in (b) for the evaluation of $L$.

would mainly affect the proportional constant, but has very little effect on the exponent.

There is a total of 717 temporal data sets of wildfire images in the database for the 31 wildfires listed in Table 1 . Each of the temporal data sets corresponds to one data point, e.g., in the plot of the burned area as a function of $R_{\mathrm{g}}$. As mentioned above, the resolution of these wildfire images is good to a scale of $5 \mathrm{~m}$. With such a high resolution and long periods of observation, one would be able to study the change in fractal dimensions as the wildfire spreads over a large area. We will study here the fractal dimensions of the burned area $\left(D_{\mathrm{f}}\right)$ and perimeter $\left(D_{\mathrm{p}}\right)$ of the recorded wildfires by using the box-counting method (Caldarelli et al., 2001). The fractal dimension $\left(D_{\mathrm{f}}\right)$ for a regular two-dimensional object such as a square is equal to 2 . We will therefore plot $2-D_{\mathrm{f}}$ vs. $R_{\mathrm{g}}$ and see how the burned areas of wildfires deviate from regular objects. Figure 4 is a plot of $2-D_{\mathrm{f}}$ vs. $R_{\mathrm{g}}$ for all the wildfires listed in Table 1 . The green dots are the data points of the longest wildfire event (Little Sand), with a total of 50 points. One can see that as $R_{\mathrm{g}}$ grows, $D_{\mathrm{f}}$ approaches 2 . The slope of a linear fit is equal to $-0.72 \pm 0.02$. This suggests that as the burned area grows larger, the perimeter of this burned area becomes smoother and closer to a regular two-dimensional object. This behavior was already observed in a percolation model studied a long time ago (Niessen and Blumen, 1986). Figure 5 is a plot of $D_{\mathrm{p}}$ vs. $R_{\mathrm{g}}$. $D_{\mathrm{p}}$ remains within the range of 1 to 1.15 as $R_{\mathrm{g}}$ grows. The average of $D_{\mathrm{p}}$ for all the data points is about 1.06 .

Figure 6 shows the result of the burned area $(A)$ as a function of $R_{\mathrm{g}}$. It is interesting to note that all the 717 data points obey an empirical function, $A \sim 4.51 R_{\mathrm{g}}^{2}$. The fact that $A$ is proportional to the square of $R_{\mathrm{g}}$ is in agreement with the empirical behavior of $D_{\mathrm{f}}$ (going to 2 as its asymptotic limit). What is more striking is the fact that they have approximately the same proportional coefficient. The error estimates of the proportional coefficient and the exponent of $R_{\mathrm{g}}$ are, respectively, $4.51 \pm 0.10$ and $2.00 \pm 0.02$. For comparison with some of the known regular two-dimensional figures, we know that the relationship between $A$ and $R_{\mathrm{g}}$ for the circle is $A=2 \pi R_{\mathrm{g}}^{2}$, while those of the square and an equilateral 


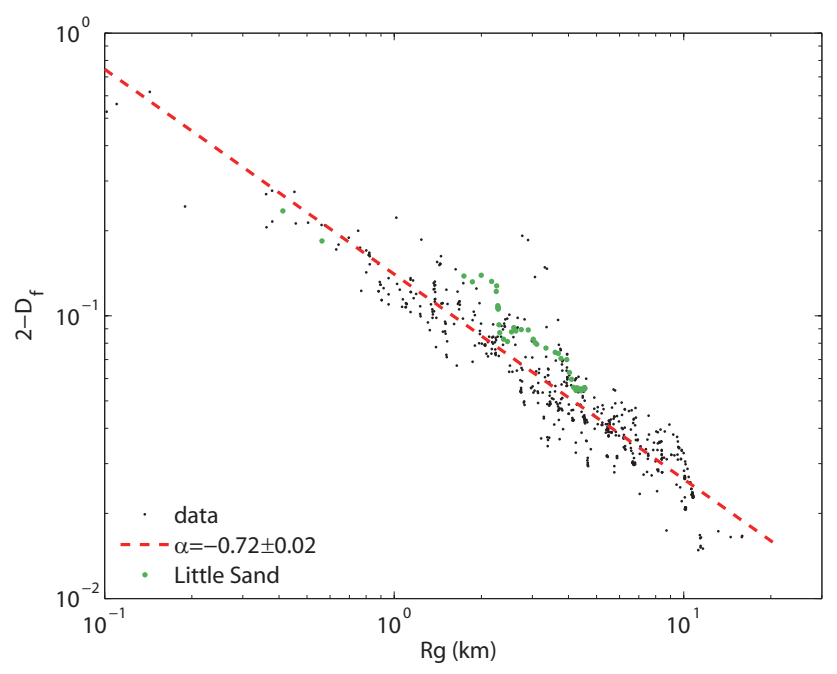

Figure 4. Plot of $2-D_{\mathrm{f}}$ vs. $R_{\mathrm{g}}$ for the 31 wildfires listed in Table 1. The green dots are the data points of the longest wildfire event (Little Sand), with a total of 50 points. The broken red line has a slope of $-0.72 \pm 0.02$.

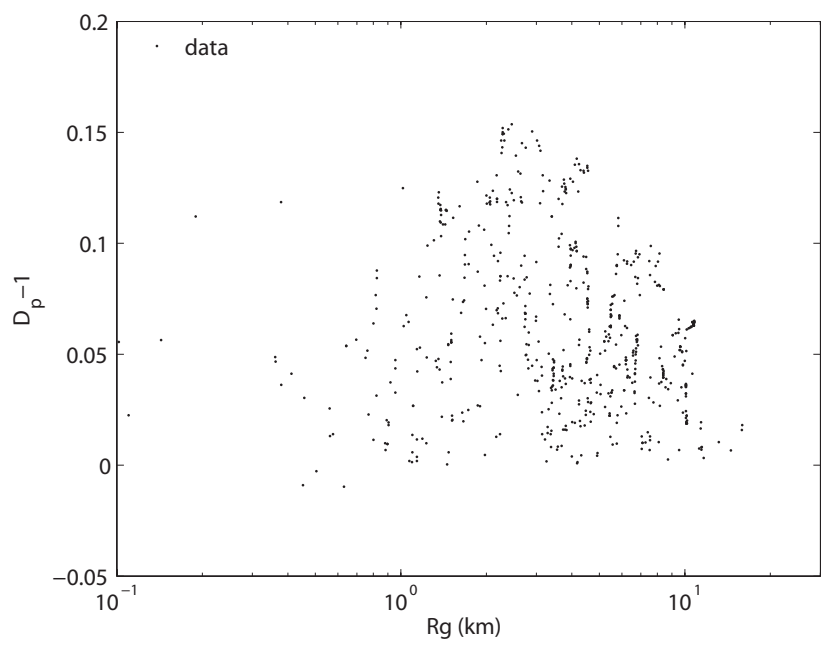

Figure 5. Plot of $D_{\mathrm{p}}$ vs. $R_{\mathrm{g}}$ for the 31 wildfires listed in Table 1.

triangle are $6 R_{\mathrm{g}}^{2}$ and $5.19 R_{\mathrm{g}}^{2}$, respectively. For a star polygon with a coefficient of $7 / 3$ heptagram (Coxeter, 1973), the coefficient is $\sim 4.49$, as shown in Fig. 7 . The result from the empirical data thus indicates that the burned areas of all the wildfires that one has recorded have shapes close to that of a two-dimensional concave polygon. Figure 8 is a plot of the boundaries $(L)$ of the wildfires as a function of $R_{\mathrm{g}}$. Again, the data points follow an empirical function, $L \sim 19.57 R_{\mathrm{g}}^{1.17}$, with an error estimate of $19.57 \pm 1.10$ and $1.17 \pm 0.04$ for the proportional coefficient and the exponent of $R_{\mathrm{g}}$, respectively.

To summarize, we have observed that both the burned area and the accessible perimeter of wildfire reveal power law behavior as a function of $R_{\mathrm{g}}$. Moreover, the burned areas of

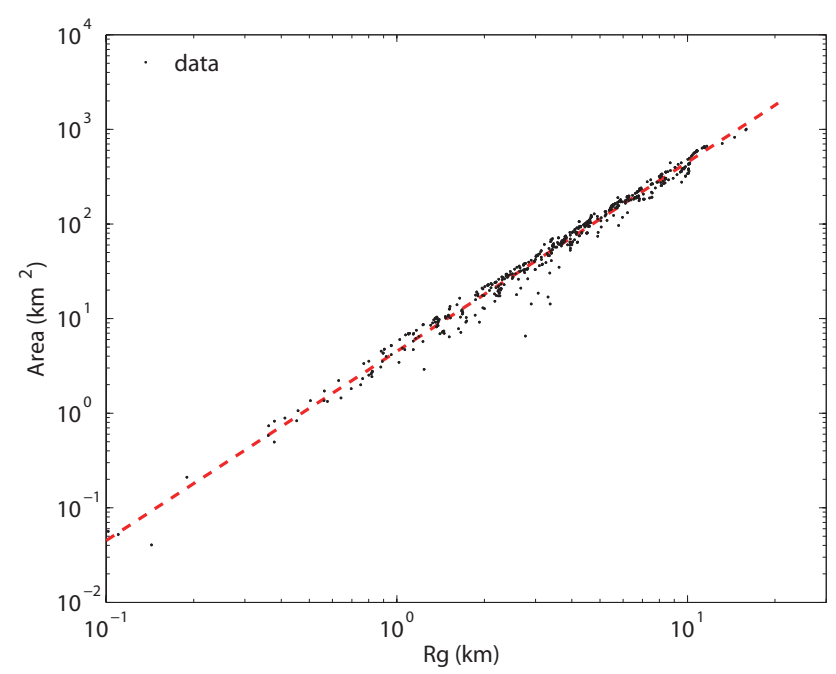

Figure 6. Burned area $(A)$ as a function of $R_{\mathrm{g}}, A \sim 4.51 R_{\mathrm{g}}^{2}$. The broken red line has a slope of 2 for comparison.

all the wildfires under study could be described by the same empirical function $A \sim 4.51 R_{\mathrm{g}}^{2}$.

\section{Model and numerical simulation}

In this section, we introduce our model for the spreading of wildfires. To try to model wildfire dynamics by taking into account all possible factors such as temperature, precipitation, wind speed, vegetation, landscape, etc. is a very complex task to achieve. In fact, a model for predicting a wildfire spreading would inevitably have to take into account these external environmental factors in a certain way. We also notice that there have already been many attempts to model wildfire spreading, ranging from physical models to empirical models (Sullivan, 2009a; Bak et al., 1990; Chen et al., 1990; Rothermel, 1972; Anderson et al., 1982). Each of these proposed models has had some success, but a complete picture of how to describe the dynamics of wildfires effectively is still not available for the moment. For our purposes, we will analyze the empirical data by employing a statistical approach. We set here an easier task by introducing a simple model to investigate the wildfire dynamics to reveal the geographic features of the wildfires under study. This is based on a cellular automata approach by introducing a two-dimensional lattice to mimic the forests that the wildfires would spread in. There exist works using a cellular automata or lattice model approach to study wildfire; see, e.g., Bak et al. (1990), Caldarelli et al. (2001), and Porterie et al. (2007). For example, people have used hexagonal cellular automata to model wildfire spreading (Trunfio, 2004; Hernandez Encinas et al., 2007; Avolio et al., 2012). There are also works that incorporate meteorological factors such as wind speed and direction, type and density of vegetation, etc. into the dynamical rules of cellular automata, and which 
a)

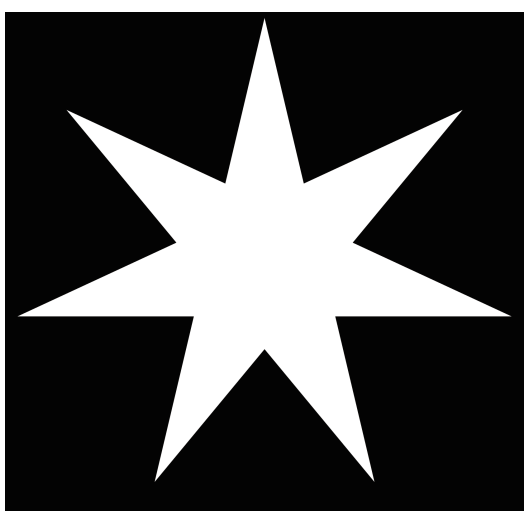

b)

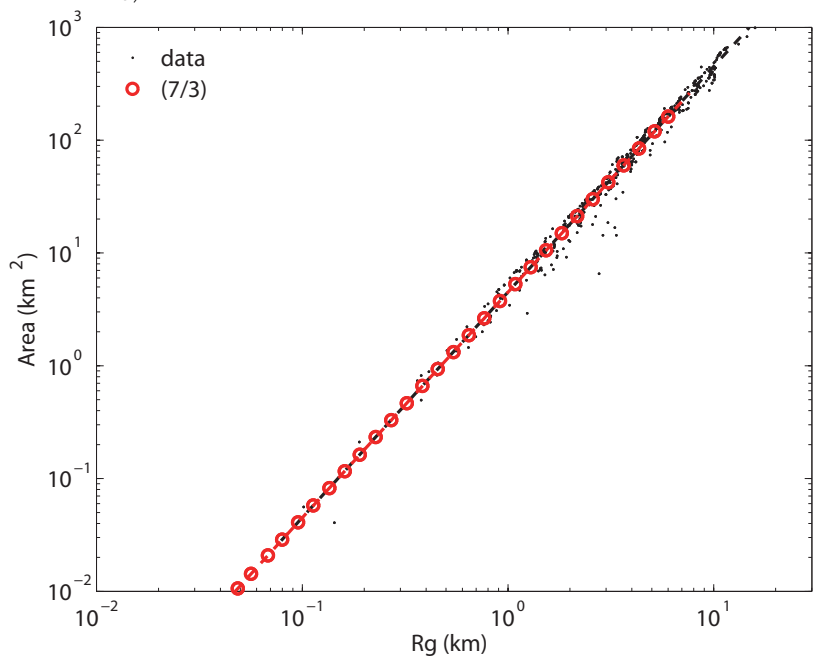

Figure 7. An illustration of a two-dimensional concave polygon, (a) a $7 / 3$ heptagon. (b) Scaling of the $7 / 3$ heptagon showed $A \sim$ $4.49 R_{\mathrm{g}}^{2}$.

compared the numerical results with the wildfire on Spetses Island in 1990 (Alexandridis et al., 2008). As a first step of our numerical study, we will not take into account these factors in the dynamical rules of our model. In this way, we are trying to capture the qualitative behavior of wildfire dynamics by using this simple model. To make it more specific, we only consider a two-dimensional square lattice here. Other types of lattices such as triangular lattices can be treated in a similar manner. On each lattice site, there is only one unit of plant, or we simply say one tree/site. In order to simulate the spreading of wildfires, we incorporate dynamical rules that can mimic the wildfires under study. The rules for the model are as follows. There are indeed three states that each site can take, namely, unburned (U), ignited (I), and burned (B). If the site is in state $\mathrm{U}$, it will remain in state $\mathrm{U}$ if it has no neighboring site in state I. In our simulation, we only consider nearest neighbors. Therefore, each lattice site will only be affected by four nearest neighbors during our simulation. On the other hand, if the site has a neighboring site in state I, it will then

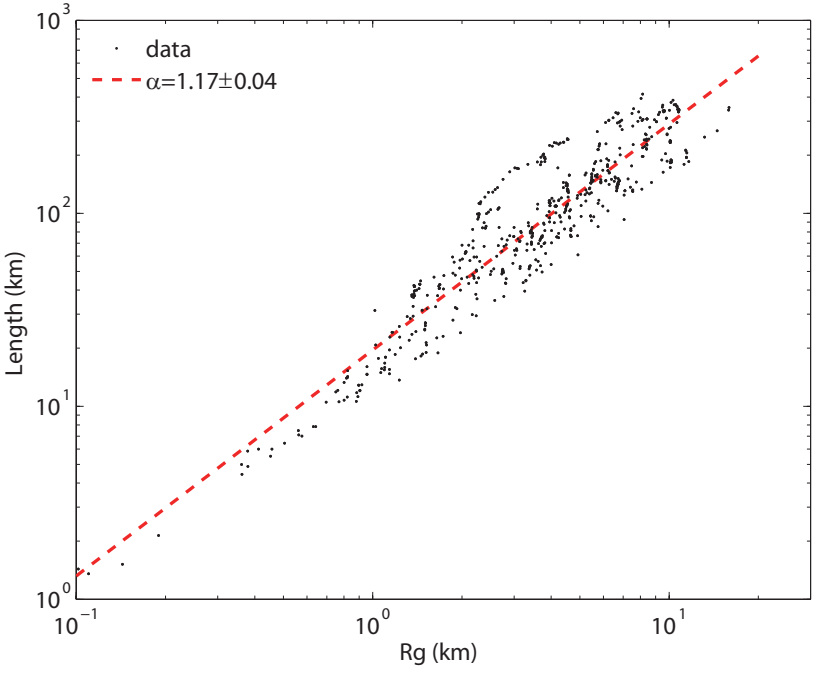

Figure 8. $L$ vs. $R_{\mathrm{g}}$ for the 31 wildfires listed in Table 1 . The broken red line is a fit of the empirical data with a slope of $1.17 \pm 0.04$.

have a certain probability $q$ that will be ignited, and if it is ignited, it will be in state I in the next time step. Furthermore, for any cell in state I, it will become burned in the next time step, i.e., in state B. Once the cell reaches state B, it will remain in state B throughout the rest of our simulation, and a burned site cannot ignite any of its neighboring sites once it reaches such a state. The rules of the spreading of wildfire at each time step $\mathrm{t}$ can now be summarized as follows: for a lattice site $i$, its state $S_{i}(t+1)$ is given by

$S_{i}(t)=\mathrm{U} \rightarrow S_{i}(t+1)=\mathrm{U}$

(no neighboring site in state I at time step $t$ )

$S_{i}(t)=\mathrm{U} \rightarrow S_{i}(t+1)=\mathrm{I}$

(a probability $q$ from each of its neighboring site in state I at time $t$ )

$\rightarrow S_{i}(t+1)=\mathrm{U}$

(a probability $1-q$ from each of its

neighboring site in state I at time $t$ )

$S_{i}(t)=\mathrm{I} \rightarrow S_{i}(t+1)=\mathrm{B}$

(the site is in state I at time $t$ )

From the above rules, one can easily see that for a site that is in state $\mathrm{U}$, and having $\mathrm{n}$ neighboring sites in state $\mathrm{I}$ at time $t$, the probability that it will be ignited at time $t+1$ is equal to $1-(1-q)^{n}$. This is the basic model that one can start with. In reality, the parameter $p$ depends on many factors, such as the climate, vegetation, landscape, etc. of the region under study. Indeed, this basic set of rules would leave too many unburned lattice sites within the burned area, which is different from what we observed in the case of real wildfires, e.g., the Little Bear wildfire shown in Fig. 1. This is illustrated in Fig. 11a, which is a sample simulation of wildfire spreading on a twodimensional square lattice with size $1024 \times 1024$ using the 


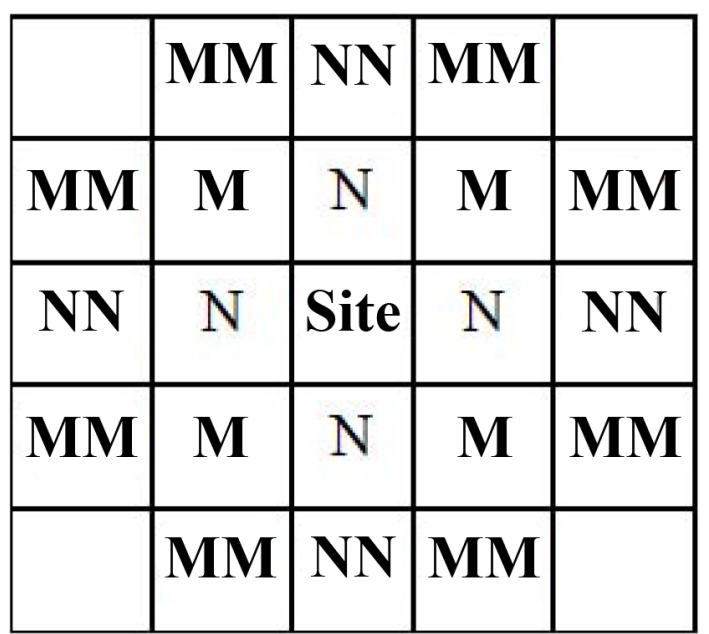

Figure 9. An illustration of the two-dimensional lattice with one burning site in the middle, with its neighbors denoted by N, M, NN and MM by their distance from the site.

above set of basic rules. We will therefore modify the dynamical rules of the model to mimic this empirical behavior. In the case of real wildfires, the threshold for the unburned plants to be on fire within the burned region could likely be lower than the area outside the burned region, thus leaving very few unburned areas within the burned region. To take this observation into account, we therefore need to introduce additional dynamical rules that could mimic such an effect. In general, it can be argued that a burning tree can ignite not only its nearest neighboring trees, but also its next neighboring trees, and sometimes even next to next-neighboring trees, etc. We therefore incorporate this scenario into our dynamical rules by taking the second, third and fourth nearest neighbors into account. This is illustrated in Fig. 9 below. The first, second, third and fourth nearest neighbors are denoted by N, $\mathrm{M}, \mathrm{NN}$ and MM, respectively. We will here choose the probability of ignition to be inversely proportional to the square of the distance between sites in the numerical simulations of the spreading of wildfires. The probability that the site would not be ignited by a neighboring burning site labeled $N$ is then equal to $(1-q)$, while the probability that it would not be ignited by a burning site labeled $M$ is equal to $(1-q)^{1 / 2}$. The overall ignition probability $f$ is

$f=1-(1-q)^{(n+m / 2+\mathrm{nn} / 4+\mathrm{mm} / 5)}$,

where $n, m, \mathrm{nn}$ and $\mathrm{mm}$ denote the number of burning sites for $\mathrm{N}, \mathrm{M}, \mathrm{NN}$ and $\mathrm{MM}$, respectively.

Another possible extension of the set of dynamical rules is to introduce into the model some kind of nonlinear effect that simulates the various factors mentioned above. We suggest here two sets of nonlinear rules, namely the fire density effect (FDE) and the burned site effect (BSE). The justification for introducing these sets of rules is the following. As the wildfire spreads, the temperature and the humidity in

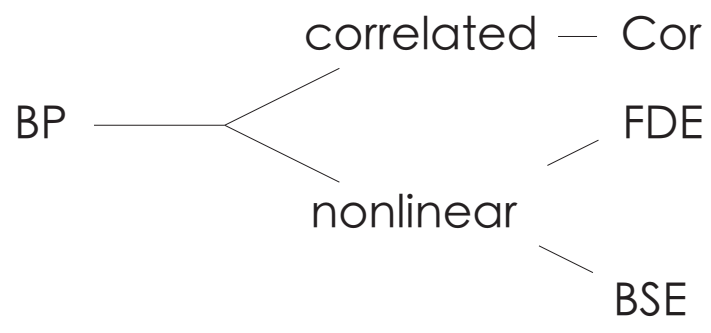

Figure 10. Set of dynamical rules used in the simulation throughout this paper. Basic percolation (BP) $f=1-(1-q)^{n}$. Fire density effect (FDE) $f=1-(1-q)^{\left(n^{k}\right)}, k \neq 1$. Burned sites effect (BSE) $f=1-(1-q)^{(n(1+z n(b)))}, z \neq 1$. Correlated (Cor) $f=1-(1-$ $q)^{(n+m / 2+n n / 4+\ldots)}$.

the neighborhood of the burning (FDE) and burned (BSE) trees will change. This would then change the probability of the unburned trees being ignited, which usually increases. To imitate these effects, we set the probability of an unburned site being ignited by FDE at $f=1-(1-q)^{\left(n^{k}\right)} \cdot k=1$ is the basic percolation model. In a similar fashion, we set the probability of an unburned site being ignited by BSE at $f=1-(1-q)^{(n(1+z n(b)))}$, where $n(b)$ denotes the number of burned sites in nearest neighbor. $z=0$ corresponds to the basic percolation. One can see that the exponent of $(1-q)$ increases as $z$ increases. This in turn means that the probability that the site will not be ignited by neighboring sites will drop faster as $z$ increases. In our simulations, we always set $z$ to be larger than or equal to 0 . Figure 10 summarizes the set of dynamical rules used in our simulation.

Figure 11 is an illustration of our simulation on a $1024 \times$ 1024 square lattice using different sets of dynamical rules in the model. As an example, we use the following set of parameters in the simulation: (a) BP, $p=0.5, q=0.38$; (b) Cor, $p=0.5, q=0.11$ (correlation length is five lattice sites); (c) FDE, $p=0.5, q=0.34, k=3$; (d) BSE, $p=0.5$, $q=0.29, z=3$. In general, the set of rules based on BSE gives better results as compared with the empirical data such as in Fig. 1.

In the remaining part of this section, we will therefore analyze statistical features of the model by performing numerical simulations using BSE rules. All simulations were performed on a $2048 \times 2048$ square lattice, with each $\mathrm{z}$ averaged over 100 simulations. In each of the simulations, we put four burning sites at the center and let the wildfire spread according to the BSE rules with different values of $z$. In performing the simulations, one can use any algorithm for asynchronous updates for the lattice. In our simulation, we use the Metroplis algorithm of the asynchronous update, which is commonly known as the Monte Carlo method in statistical physics (Metropolis et al., 1953). Figure 12 is an illustration of the spreading of burned areas with BSE for different values of $z$. One can see that as $z$ increases, so does the spreading of burned areas. Note that for $z \leq 1$, the burned 


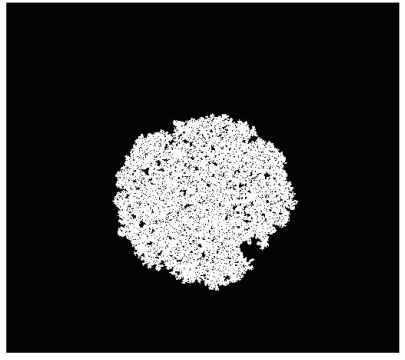

(a)

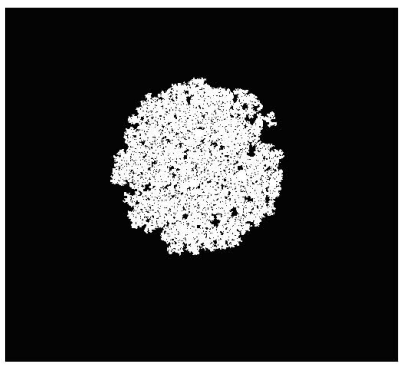

(c)

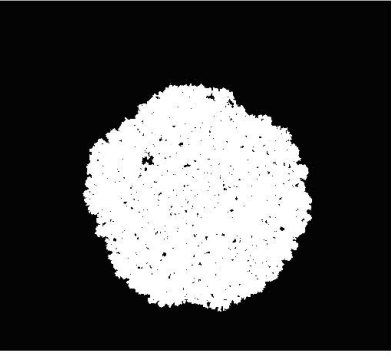

(b)

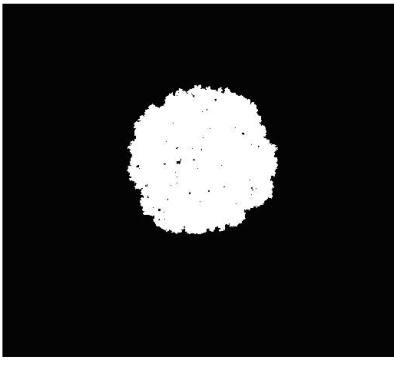

(d)
Figure 11. (a) $\mathrm{BP}, p=0.5, q=0.38$; (b) Cor, $p=0.5, q=0.11$ (correlation length is five lattice sites); (c) FDE, $p=0.5, q=0.34$, $k=3$; and (d) BSE, $p=0.5, q=0.29, z=3$.

area stops growing after a few hundred Monte Carlo steps. The result also shows that the burned area for the same number of Monte Carlo steps increases rapidly as z increases. In performing the numerical simulation, one Monte Carlo step usually refers to one sweep of updates for the lattice; i.e., if the lattice is an $N \times N$ lattice, there will be an average $N^{2}$ update for each Monte Carlo step. In our simulation here, one only needs to perform updates for the burning sites and their neighboring sites in each Monte Carlo step. Furthermore, since one only performs about 1000 Monte Carlo steps for each wildfire spread in Fig. 12, one can employ any type of boundary condition on the lattice, since the burned sites will never get to the boundaries of the lattice. Note that we also include the empirical data points in Fig. 12 for comparison. In general, one would consider a forest to have about 10 trees or more for each hectare $\left(10000 \mathrm{~m}^{2}\right)$. We therefore assume that the distance between two adjacent lattice sites is equivalent to a separation of $25 \mathrm{~m}$. Figure 13 presents the numerical results of $L$ vs. $R_{\mathrm{g}}$ with BSE for different values of $z$. One can see that there is a gradual decrease in $\beta\left(L \propto R_{\mathrm{g}}^{\beta}\right)$ as $z$ increases.

Figure 14 shows $D_{\mathrm{p}}$ vs. $R_{\mathrm{g}}$, with BSE for different values of $z$. When the simulated burned area is small, the perimeter is more rugged, and the increase in the burned area does not change the perimeter as fast as a regular two-dimensional object. As the burned area increases, so does $D_{\mathrm{p}}$, and it will eventually approach an asymptotic value close to that of a regular two-dimensional object. a)

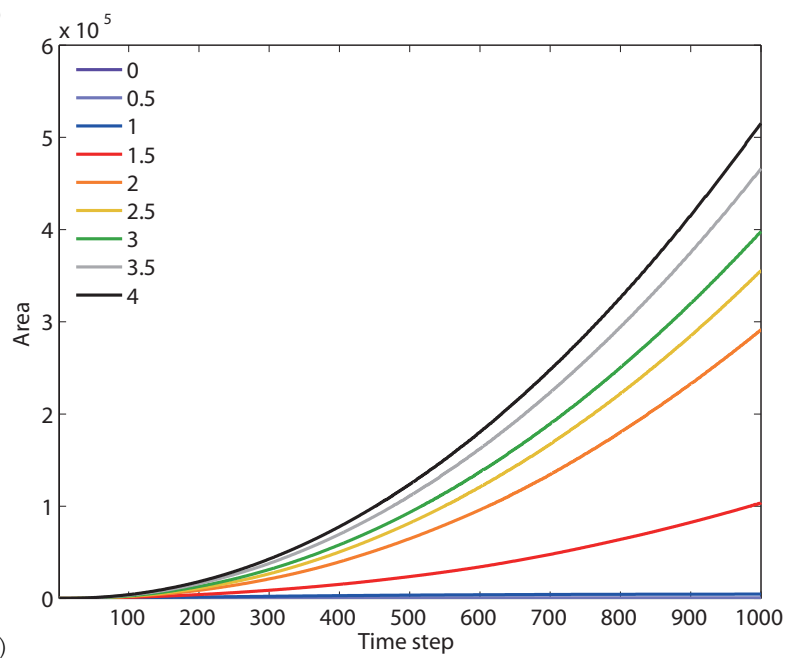

b)

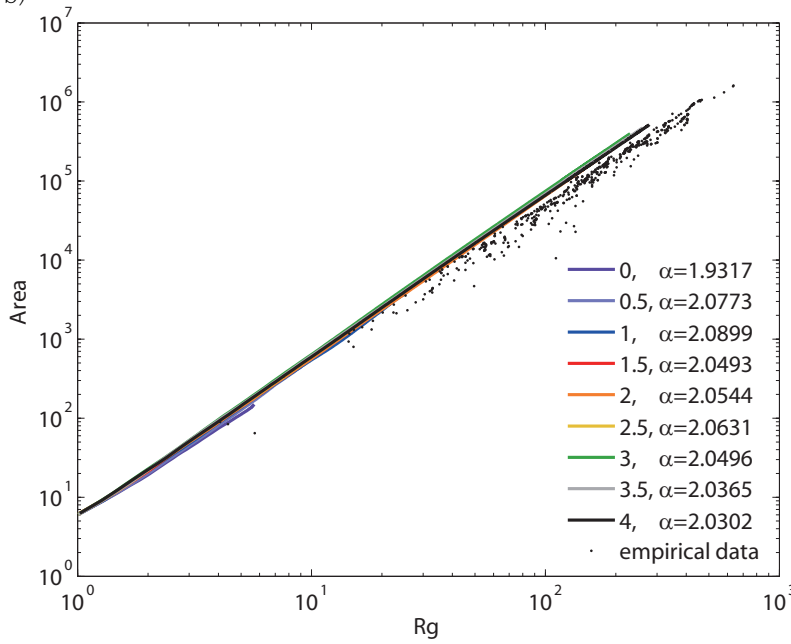

Figure 12. (a) The spreading of burned areas with BSE for different values of $z$. The burned area for the same number of Monte Carlo steps increases rapidly as $z$ increases (changing the labeling of the steps). (b) The corresponding result of the burned area as a function of $R_{\mathrm{g}}$, with BSE and fitting $A \propto R_{\mathrm{g}}^{\alpha}$. Empirical data points are also included for comparison.

Note that in Figs. 12-14, $R_{\mathrm{g}}$ is measured in terms of the lattice length unit (one unit is the distance between two neighboring lattice sites), while the area $A$ is measured in terms of the number of unit lattice cells.

\section{Conclusions}

In this paper, we have analyzed time series data of 31 wildfires in the US that occurred in 2012, with a total of 717 data sets of wildfire images. With a spatial resolution of $5 \mathrm{~m}$ and daily data for these wildfire incidents, one is able to carry out a better analysis of the dynamical and statistical properties of wildfires. The behavior of fractal dimensions (FD) during the spreading was studied and their geological features were identified. The result shows that the fractal 


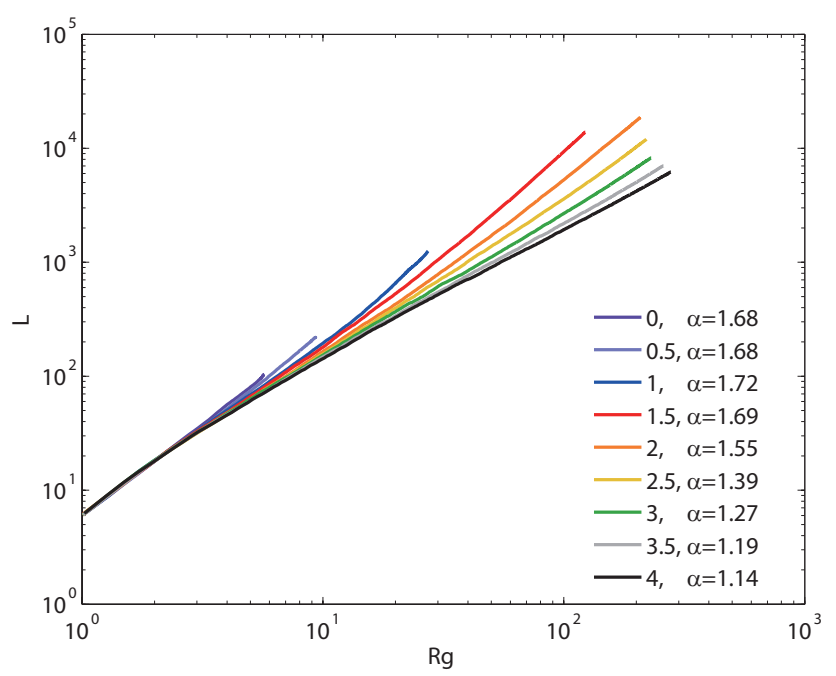

Figure 13. $L$ vs. $R_{\mathrm{g}}$, with BSE for different values of $z$.

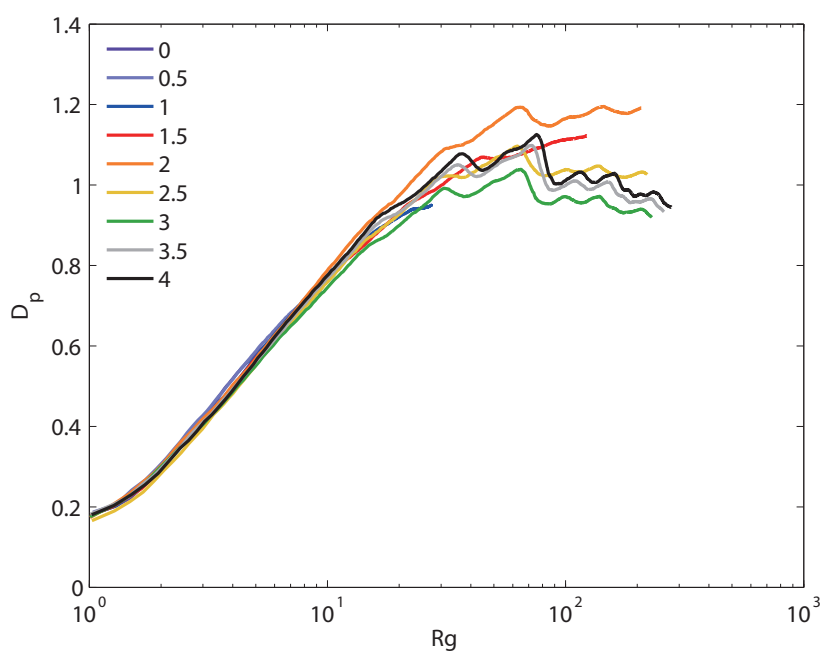

Figure 14. $D_{\mathrm{p}}$ vs. $R_{\mathrm{g}}$, with BSE for different values of $z$.

dimension $D_{\mathrm{f}}$ of the burned areas approaches 2 , while $D_{\mathrm{p}}$ of the corresponding accessible perimeter ranges between 1 and 1.15 as the wildfire spreads. More interestingly, all data points follow empirical relations for the burned area $\left(A \sim 4.51 R_{\mathrm{g}}^{2}\right)$ and accessible perimeter $\left(L \sim 19.57 R_{\mathrm{g}}^{1.17}\right)$. In a spatially uniform landscape, the fractal dimension of wildfire would be 2 . The observation that the fractal dimension of wildfire spreading is less than 2 could mean that its dynamics is driven by spatial heterogeneity in the environment, e.g., by different types of plants, different distances between patches, etc., which may reduce the spatial spreading of wildfire. This may well imply that the fractal dimension of wildfires observed in the data is a proxy for spatial heterogeneity, which may come about because of differences in environmental, climatic and other factors between locations. The fact that wildfire spreading exhibits fractal behavior suggests that the underlying dynamics can be characterized by the fractal structures. One can thus study models whose dynamics incorporate such fractal structures without knowing the details of effects from ecology, climate, etc. as a first step to understanding the dynamics of wildfire spreading. Taking this as a starting point, a growth model based on the cellular automata method is proposed in this paper to mimic both the dynamical and statistical properties of the spreading of wildfires. Numerical results are compared with the fractal dimensions and scaling behaviors of the corresponding empirical data, and show good agreement between the simulated data from the model and the empirical data from the database (Geomac, 2012). The results suggest that the simple model proposed here is able to capture the qualitative behavior of wildfire dynamics. It would be interesting to see if one can further extend the model to incorporate the meteorological factors as mentioned above.

Edited by: A. Ganguly

Reviewed by: two anonymous referees

\section{References}

Alexandridis, A., Vakalis, D., Siettos, C. I., and Bafas, G. V.: A cellular automata model for forest fire spread prediction: The case of the wildfire that swept through Spetses Island in 1990, Appl. Math. Comput., 204, 191-201, 2008.

Anderson, D. G., Catchpole, E. A., DeMestre, N. J., and Parkes, E.: Modeling the spread of grass fires, J. Austral. Math. Soc., Series B, 23, 451-466, 1982.

Avolio, M. V., Di Gregorio, S., Spataro, W., and Trunfio, G. A.: A theorem about the algorithm of minimization of differences for multicomponent cellular automata, Lect. Not. Comp. Sc., 7495, 289-298, 2012.

Bak, P., Chen, K., and Tang, C.: A forest-fire model and some thoughts on turbulence, Phys. Lett. A, 147, 297-300, 1990.

Caldarelli, G., Frondoni, R., Gabrielli A., Montuori, M., Retzlaff, R., and Ricotta, C.: Percolation in real wildfires, Europhys. Lett., 56, 510, doi:10.1209/epl/i2001-00549-4, 2001.

Chen, K., Bak, P., and Jensen, M. H.: A deterministic critical forest fire model, Phys. Lett. A, 149, 207-210, 1990.

Coxeter, H. S. M.: Regular polytopes, Dover Publications, NY, 1973.

Hernandez Encinas, L., Hoya White, S., Martin del Rey, A., and Rodriguez Sanchez, G.: Modelling forest fire spread using hexagonal cellular automata, Appl. Math. Model., 31, 1213-1227, 2007.

Geomac, http://www.geomac.gov/index.shtml (last access: July 2014), 2012.

Kitzberger, T., Brown, P. M., Heyerdahl, E. K., Swetnam, T. W., and Veblen, T. T.: Contingent Pacific-Atlantic Ocean influence on multicentury wildfire synchrony over western North America, P. Natl. Acad. Sci. USA, 104, 543-548, 2007.

Lee, H.-I., Wang, S.-L., and Li, S.-P.: Climate Effect on Wildfire Burned Area in Alberta (1961-2010), Int. J. Mod. Phys. C, 24, 1350053, doi:10.1142/S0129183113500538, 2013.

Littell, J. S., McKenzie, D., Peterson, D. L., and Westerling, A. L.: Climate and Ecoprovince Fire Area Burned in Western U.S. Ecoprovinces, 1916-2003, J. Appl. Meteor. Climatol., 19, 10031021, 2009. 
Matthew, S. G., Platt, W. J., Beckage, B., Orzell, S. L., and Taylor, W.: Accurate quantification of Seasonal Rainfall and Associated Climate-Wildfire Relationships, J. Appl. Meteor. Climatol., 49, 2559-2573, 2010.

Mckenzie, D., Peterson, D. L., and Alvarado, E.: Extrapolation problems in modeling fire effects at large spatial scales, Int. J. Wildland Fire, 6, 165-176, 1996.

Metropolis, N., Rosenbluth, A. W., Rosenbluth, M. N., Teller, A. H., and Teller, E.: Equation of State Calculations by Fast Computing Machines, J. Chem. Phys., 21, 1087, doi:10.1063/1.1699114, 1953.

Niessen, W. V. and Blumen, A.: Dynamics of forest fires as a directed percolation model, J. Phys. A, 19, L289, doi:10.1088/0305-4470/19/5/013, 1986.

Porterie, B., Zekri, N., Clerc, J. P., and Loraud, J. C.: Modeling forest fire spread and spotting process with small world networks, Comb. Flame, 149, 12, 63-78, 2007.

Romme, W. H.: Fire and landscape diversity in subalpine forests of Yellowstone National Park, Ecol. Monogr. 52, 199-221, 1982.

Rothermel, R. C.: A mathematical model for predicting fire spread in wildland fuels, Intermountain Forest and Range Experiment Station, Forest Service, US Department of Agriculture, INT 115 Odgen, Utah, USA, 1972.
Sullivan, A. L.: Wildland surface fire spread modelling, 1990-2007. 1: Physical and quasi-physical models, Int. J. Wildland Fire, 18, 349-368, 2009a.

Sullivan, A. L.: Wildland surface fire spread modelling, 1990-2007. 2: Empirical and quasi-empirical models, Int. J. Wildland Fire, 18, 369-386, 2009b.

Sullivan, A. L.: Wildland surface fire spread modelling, 1990-2007. 3: Simulation and mathematical analogue models, Int. J. Wildland Fire, 18, 387-403, 2009c.

Trunfio, G. A.: Predicting Wildfire Spreading through a Hexagonal Cellular Automata Model, Lect. Not. Comp. Sc., 3305, 385-394, 2004.

Weise, D. and Biging, G. S.: Effects of wind velocity and slope on flame properties, Can. J. Forest Res., 26, 1849-1858, 1996.

Westerling, A. L. and Bryant, B. P.: Climate Change and Wildfire in California, Clim. Change, 87, 231-249, 2008.

Westerling, A. L., Hidalgo, H. G., Cayan, D. R., and Swetnam, T. W.: Warming and Earlier Spring Increase Western U.S. Forest Wildfire Activity, Science, 313, 940-943, 2006. 